\title{
Alternativas de controle de doenças de pós-colheita em citros
}

\author{
Vanessa Santos Moura ${ }^{1}$, Rafael Kupper Moretto ${ }^{2}$, Bianca Ikari Machado ${ }^{3}$ \& \\ Katia Cristina Kupper ${ }^{4 *}$
}

\begin{abstract}
RESUMO
O Brasil destaca-se como maior produtor de laranja do mundo, exportando 372.380 toneladas dessa fruta na safra 2017/2018. O cultivo de citros é responsável pela criação de mais de 45 mil novos postos de trabalho. Apesar da importância econômica e social da citricultura para o país, o setor vem enfrentando vários problemas fitossanitários que limitam a sua produção, dentre esses, a ocorrência de doenças que ocorrem na fase de pós-colheita. Os bolores verde e azul, causados por Penicillium digitatum e P. italicum, respectivamente, e a podridão azeda, causada por Geotrichum citri-aurantii, são as enfermidades mais importantes, em termos econômicos, pois afetam não só a quantidade, como a qualidade dos frutos cítricos, depreciando-os para o comércio in natura. Considerando as preocupações dos consumidores com o meio ambiente e com a saúde pública, devido ao uso excessivo de fungicidas, além da falta de fungicida registrado para controle da podridão azeda em citros, este estudo teve por objetivo fornecer informações sobre alternativas para o controle de doenças que ocorrem na pós-colheita de frutos cítricos, como o uso de microrganismos antagônicos, produtos naturais e termoterapia. Resultados apresentados neste estudo mostram o potencial dos métodos alternativos de controle, sozinhos ou em combinação, de doenças que ocorrem na pós-colheita de citros.
\end{abstract}

Termos de indexação: Bacillus spp., leveduras, quitosana, extratos vegetais, termoterapia.

\section{Alternative for the control of postharvest citrus diseases}

\section{SUMMARY}

Brazil stands out as the largest orange producer in the world, where the 2017/2018 harvest exported 372,380 tons and was responsible for the creation of more than 45 thousand new jobs. Despite the economic and social importance of citriculture to the country, the sector has been facing several phytosanitary problems that limit its production, among them, the occurrence of diseases that occur in the post-harvest phase. The green and blue molds caused by Penicillium digitatum and P. italicum, respectively, and the sour rot caused by Geotrichum citri-aurantii are the most

\footnotetext{
${ }^{1}$ Programa de Pós-graduação em Microbiologia Agropecuária, Universidade Estadual Paulista "Júlio de Mesquita Filho" UNESP, Jaboticabal, SP, Brasil

${ }^{2}$ Programa de Pós-graduação em Produção Vegetal e Bioprocessos Associados, Universidade Federal de São Carlos - UFSCar, Araras, SP, Brasil

${ }^{3}$ Programa de Pós-graduação em Agroecologia e Desenvolvimento Rural, Universidade Federal de São Carlos - UFSCar, Araras, SP, Brasil

${ }^{4}$ Centro Avançado de Pesquisa de Citros "Sylvio Moreira”, Instituto Agronômico - IAC, Cordeirópolis, SP, Brasil.

Autor correspondente: Centro Avançado de Pesquisa de Citros "Sylvio Moreira", Instituto Agronômico - IAC, CEP 13490-970, Cordeirópolis, SP, Brasil. E-mail de correspondência: katia@ccsm.br
} 
important diseases in economic terms, since they affect not only the quantity and the quality of the citrus fruits, depreciating them for in natura trade. Considering the concerns of consumers with the environment and public health, due to excessive and indiscriminate use of some fungicides, in addition to the lack of fungicide registered to control sour rot in citrus, this study aimed to provide information on alternatives such as the use of antagonistic microorganisms, natural products and thermotherapy for the control of diseases that occur in the post-harvest of citrus fruits. Results presented in this study show the potential of alternative methods for the control, alone or in combination, of diseases that occur in the post-harvest of citrus.

Index terms: Bacillus spp., yeasts, chitosan, vegetables extracts, thermotherapy.

\section{INTRODUÇÃO}

A cadeia citrícola representa um importante segmento agroindustrial para a economia brasileira, devido à demanda econômica gerada pela comercialização dos frutos. Além de promover divisas econômicas, o setor tem grande impacto na geração de empregos, de forma direta e indireta, contribuindo para a balança comercial nacional, tanto na geração de capital, quanto no desenvolvimento regional (Lopes, 2009; Zulian et al., 2013).

O Brasil destaca-se como maior produtor de laranja do mundo, sendo representativo no estado de São Paulo e Triangulo mineiro, compreendendo 12 mil fazendas ativas, gerando um PIB de US\$ 6,5 bilhões de dólares em todos os elos da cadeia produtiva. A safra 2017/2018 exportou 372.380 toneladas, sendo responsável pela criação de 45.508 novos postos de trabalho no interior paulista (Neves \& Trombin, 2017).

Apesar da importância econômica e social da citricultura para o país, o setor vem enfrentando vários problemas fitossanitários com a ocorrência de pragas e doenças que limitam a sua produção. Segundo Kupper et al. (2016), existem em torno de 21 doenças provocadas por bactérias, fungos e vírus que acarretam em danos econômicos bastante expressivos. A aplicação de produtos químicos nos pomares, ou durante o beneficiamento dos frutos, ainda é o método mais utilizado para o controle da maioria das doenças de citros (Spadaro \& Droby, 2016).

Com relação às doenças que ocorrem na fase de pós-colheita dos citros, os bolores verde e azul, causados por Penicillium digitatum Sacc e P. italicum Wehner, respectivamente, e a podridão azeda, causada por Geotrichum citri-aurantii (Ferraris) R. Cif. \& F. Cif. (Sinônimo G. candidum Link) (Pitt \& Hocking, 1997) são as mais importantes, em termos econômicos, pois afetam não só a quantidade como a qualidade dos frutos cítricos, depreciando-os para o comércio in natura.

O controle dos bolores em galpão de embalagem baseia-se no tratamento de frutos com fungicidas, como imazalil (IMZ) e tiabendazol (TBZ). Estes fungicidas são utilizados sozinhos, em misturas, ou aplicados separadamente, em sequencia, sendo os mesmos utilizados há mais de 25 anos, o que contribui para a proliferação de linhagens resistentes dos fungos aos princípios ativos utilizados (Ismail \& Zhang, 2004).

A podridão azeda é descrita em todos os países produtores, afetando todas as espécies e cultivares de citros (Talibi et al., 2012). Atualmente, a doença tem preocupado mais os agricultores, pois os prejuízos já estão ocorrendo na pré-colheita dos citros, especialmente durante os períodos chuvosos (Liu et al., 2009; Feng et al., 2011), e continuam durante o beneficiamento dos frutos. Para o controle, os fungicidas guazatina e propiconazole vêm sendo utilizados em vários países da Europa, África do Sul e Austrália (Kellerman et al., 2018; McKay et al., 2012; Ismail \& Zhang, 2004), porém esses produtos não possuem registros no Brasil. De acordo com Hao et al. (2010), a falta de fungicidas registrados é um problema sério e um desafio na busca de estratégias efetivas e seguras para o controle da doença.

Cuidados adotados na colheita, manuseio e transporte dos frutos, de modo a reduzir os ferimentos causados na superfície dos frutos, podem diminuir os riscos de infecções (Johnson \& Heather, 1995), assim como, a adoção de práticas culturais, como nutrição das plantas adequada, podas, remoção do inóculo, colheita das frutas nos estádios adequados de maturação, além dos cuidados durante o beneficiamento das frutas com manuseio, temperatura, umidade relativa, atmosfera controlada, termoterapia e a quimioterapia (Cappellini \& Ceponis, 1984). No entanto, o uso de produtos químicos ainda é a forma mais eficiente de controle de algumas doenças de pós-colheita de citros (Benato, 1999; Zambolim, 2002).

A aplicação de estratégias alternativas de controle visa não só manter a alta produtividade, mas também, diminuir os impactos negativos gerados pelo uso frequente e excessivo de produtos químicos para o controle de doenças na pós-colheita dos citros. Considerando a falta 
de produto registrado para controle da podridão azeda em citros, as preocupações com o meio ambiente e com a saúde pública, com o uso excessivo de fungicidas para o controle de bolores, este estudo teve por objetivo fornecer informações sobre alternativas, como o uso de microrganismos antagônicos, produtos naturais e termoterapia para o controle de doenças que ocorrem na pós-colheita de frutos cítricos.

\section{Microrganismos como agentes de controle biológico}

O controle biológico é uma importante estratégia de manejo para doenças fúngicas, tendo como princípio básico a utilização de microrganismos antagonistas para reduzir e/ou manter a população de um fitopatógeno abaixo dos níveis que causam perda econômica (Carmona-Hernandez et al., 2019).

Microrganismos antagônicos podem suprimir, ou interferir no crescimento e na atividade patogênica de fitopatógenos, sendo os mesmos referenciados como "agentes de controle biológico" (Dukare et al., 2018). Nos últimos anos, o controle biológico na pós-colheita de frutos vem se mostrando como uma alternativa promissora ao controle químico (Zhimo et al., 2016).

Em geral, os mecanismos de ação dos microrganismos antagônicos no biocontrole são competição por espaço e nutrientes; competição pelo elemento ferro; produção de compostos antifúngicos e compostos voláteis; parasitismo; produção de enzimas hidrolíticas; indução de resistência; resposta oxidativa e produção de toxina killer (Liu et al., 2013; Ferraz et al., 2016; Spadaro \& Droby, 2016; Cunha et al., 2018). Portanto, compreender esses mecanismos é fundamental quando se busca a produção de um bioproduto.

Segundo Spadaro \& Droby (2016), os produtos de biocontrole disponíveis para uso na pós-colheita ainda são limitados, devido à redução e inconsistência do desempenho dos agentes de biocontrole (ACBs), bem como a resistência oferecida pelo mercado e o tamanho reduzido de empresas envolvidas no desenvolvimento e comercialização dos bioprodutos.

O gênero Bacillus vem sendo utilizado como agente de controle biológico de fungos causadores de doenças em plantas, inclusive na pós-colheita (Kupper et al., 2013), devido à sua capacidade de formar endósporos, o que facilita o desenvolvimento de produtos mais estáveis e viáveis, podendo, ainda, serem usados para a promoção de crescimento em plantas (Sanders et al., 2003).
A atividade antagônica de espécies de Bacillus pode estar associada com a produção de antibióticos (Kupper \& Fernandes, 2002; Junior et al., 2017), além da produção de voláteis antifúngicos (Pinchuk et al., 2002; Leelasuphakul et al., 2008; Fujimoto \& Kupper, 2016). Segundo alguns autores, antibióticos produzidos por bactérias dos gêneros Bacillus e Pseudomonas foram os responsáveis pelo antagonismo contra patógenos que ocorrem na pós-colheita (Dukare et al., 2018).

Fujimoto \& Kupper (2016), ao estudarem diferentes isolados de Bacillus spp., verificaram que os metabólitos produzidos pela bactéria foram capazes de inibir o crescimento de Phyllosticta citricarpa, mantendo suas atividades antagônicas mesmo após os mesmos serem submetidos à alta temperatura $\left(120^{\circ} \mathrm{C}\right)$.

Hong et al. (2014) estudaram o potencial de um isolado de Bacillus amyloliquefaciens HF-01 sozinho, ou em combinação com bicarbonato de sódio, e o tratamento térmico contra os fungos $P$. digitatum, $P$. italicum e G. citri-aurantii. Os autores verificaram que a bactéria combinada com $2 \%$ de bicarbonato de sódio e/ou tratamento térmico $\left(45^{\circ} \mathrm{C}\right.$ durante $2 \mathrm{~min}$ ) foi tão eficaz quanto o tratamento com o produto químico, reduzindo a doença em $80 \%$, em comparação com o tratamento testemunha, não prejudicando a qualidade dos frutos armazenados a $25^{\circ} \mathrm{C}$ por 4 semanas, ou a $6{ }^{\circ} \mathrm{C}$ por 8 semanas.

As leveduras, como agentes de controle biológico de doenças de pós-colheita, são potencialmente efetivas, uma vez que são hábeis na colonização de ferimentos, possuem baixa mico-toxicidade, são exclusivamente adaptadas ao microambiente de frutas (alta concentração de açúcar, alta pressão osmótica e baixo $\mathrm{pH}$ ), e podem ser tolerantes aos fungicidas frequentemente utilizados na pós-colheita (Filonow, 1998; Coelho et al., 2003; Lahlali et al., 2011; Spadaro \& Droby, 2016).

Ferraz et al. (2016), ao estudarem oito isolados de leveduras, verificaram que os isolados ACB-K1 - Saccharomyces cerevisiae, ACBL-23 - Rhodotorula minuta, ACBL-44 - Candida azyma e ACBL-77 - Aureobasidium pullulans apresentaram potencial para o controle da podridão azeda em frutos cítricas, tanto preventivamente, quanto curativamente. $\mathrm{O}$ isolado $\mathrm{ABC}-\mathrm{K} 1$ produziu duas classes de enzimas hidrolíticas (quitinases e $\beta$-1,3-glucanases), além de apresentar atividade killer contra o fitopatógeno. Nesse estudo, os autores mostraram, ainda, a eficiência de um isolado de Sporobolomyces koalae no biocontrole da podridão azeda, sendo o primeiro relato dessa levedura como um agente de controle biológico. 
Klein \& Kupper (2018) verificaram que a adição de sulfato de amônio (1\%), como fonte de nitrogênio em meio batata-dextrose-ágar para cultivo da levedura, estimulou a produção de biofilme pelo microrganismo, aumentando a atividade antagônica contra o fungo G. citri-aurantii, sendo este o primeiro relato de uma correlação positiva entre o aumento na quantidade de biofilme produzido por A. pullulans, com o aumento da atividade antagônica.

Medina-Córdova et al. (2018) demonstraram o potencial de Debaryomyces hansenii em minimizar a presença do bolor azul nos frutos de limas e da podridão azeda em uvas, enquanto que Moretto et al. (2014) relataram um controle de $100 \%$ do bolor verde ( $P$. digitatum), quando frutas de lima ácida Tahiti foram tratadas com meia dose de imazalil associada com a levedura Saccharomyces cerevisiae.

Alguns produtos à base de leveduras já são comercializados, como a formulação a base de Metschnikowia fructicola, produto denominado Shemer ${ }^{\circledR}$ e utilizado comercialmente em Israel para o controle de doenças pós-colheita de batata-doce, uva, morango, pimenta e cenoura (Droby et al., 2009; Kurtzman \& Droby, 2001). $\mathrm{Na}$ Espanha, estão disponíveis os bioprodutos Pantovital ${ }^{\circledR}$ (Pantoea agglomerans) e Candifruit ${ }^{\mathrm{TM}}$ (Candida sake) (Usall et al., 2009). Produtos como Aspire ${ }^{\circledR}$ (Ecogen Inc., Langhorn, PA) e YieldPlus ${ }^{\circledR}$ (Anchor Yeast, Cape Town, South Africa), a base de Candida oleophila e Cryptococcus albidus, respectivamente, foram os pioneiros para o controle de doenças pós-colheita em citros, porém, não estão mais disponíveis no mercado (Maia, 2011), devido às deficiências comerciais relacionadas ao marketing (Carmona-Hernandez et al., 2019). O bioproduto Boni-Protect $^{\circledR}$, a base da combinação de duas cepas de A. pullulans, apresenta registro na Alemanha para controle de Monilia fructigena, P. expansum e B. cinerea em maçã (Wagner et al., 2013).

As vantagens com relação à utilização de produtos à base de microrganismos são inúmeras, dentre essas, a redução de contaminantes ou de resíduos químicos nas superfícies dos alimentos (Luo et al., 2019); a segurança quanto à aplicação desses produtos, com menores riscos à saúde pública e ao meio ambiente (Droby et al., 2002), com probabilidade de diminuir drasticamente a contaminação do solo e da água, sendo portanto, uma alternativa ecologicamente correta no controle de doenças, não só da pós-colheita, como também, das doenças que ocorrem na pré-colheita e, que muitas vezes podem se manifestar na pós-colheita. Por outro lado, o desenvolvimento de um bioproduto é um processo complexo, longo, muitas vezes pode ser dispendioso e a sua eficácia nem sempre pode ser comparada aos fungicidas convencionais (Obagwu $\&$ Korsten, 2003). A falta de eficácia muitas vezes ocorre pelo fato dos produtos dependerem de fatores ambientais, que nem sempre são possíveis de controle, como temperatura, umidade, precipitação e, assim por diante, aspectos abióticos que os fungicidas sintéticos conseguem superar por serem moléculas com maior estabilidade quando comparado aos microrganismos formulados (Nunes, 2012).

\section{Produtos naturais}

Entre as medidas alternativas para o manejo de doenças pós-colheita em citros está o uso de produtos naturais, provenientes de extratos de plantas com propriedades antibióticas contra fitopatógenos (Senhor et al., 2009).

A quitosana é um polissacarídeo de alta massa molecular, solúvel em ácidos orgânicos, comestível e seguro para os seres humanos (Bautista-Banõs et al., 2006). Segundo Dutta et al. (2007), esse produto oferece um promissor e versátil polímero biodegradável para embalagens de alimentos, com potencial para prolongar a vida de armazenamento e controlar as podridões em várias culturas.

A quitosana tem apresentado efeito quando aplicada sozinha em morangos, no controle pós-colheita dos fungos Botrytis cinerea, Rhizopus stolonifer e Penicillium spp., responsáveis pelas doenças mofo cinzento, podridão mole e bolores, respectivamente (Romanazzi et al., 2013). Esse produto natural tem se mostrado efetivo para o controle de doenças devido à capacidade para formar filmes protetores, similares às ceras empregadas no revestimento de frutas e, assim, atuar na regulação das trocas de gases e de umidade entre o produto e o ambiente, além da possibilidade de induzir respostas de resistência nos tecidos vegetais (El-Ghaouth et al., 1992). Segundo Terry \& Joyce (2003), estes efeitos têm sido atribuídos à sua atividade direta antifúngica, ao efeito da modificação atmosférica e/ou a indução na pós-colheita de respostas de resistências em tecidos vegetais.

Vários trabalhos relataram que o crescimento micelial de fungos fitopatogênicos pode ser inibido ou retardado com a adição de quitosana ao meio de cultivo. Segundo El-Ghaouth et al. (1992), com o aumento da concentração de quitosana $(0,75-6 \mathrm{mg} / \mathrm{mL})$, o crescimento radial de Alternaria alternata, Botrytis cinerea, Colletotrichum gloeosporioides e Rhizopus stolonifer foi diminuído. 
O mesmo efeito foi observado para Sclerotinia sclerotiorum, com o aumento das concentrações de quitosana de 1 a $4 \%$ (Cheah et al., 1997).

A combinação de glicolquitosano $(0,2 \%)$ com a levedura antagonista Candida saitoana; para o controle de doenças pós-colheita, demostrou alto nível de proteção em citros e maçã, contra Penicillium digitatum e $P$. expansum, respectivamente, alcançando controle sendo significativamente maior que o obtido para cada tratamento separadamente (El-Ghaouth et al., 2000).

Estudos recentes demonstraram que a quitosana não é apenas eficaz na diminuição do crescimento do fitopatógeno, mas pode, também, causar alterações morfológicas e desorganizações moleculares das células fúngicas (El-Ghaouth et al., 1999; Ait Barka et al., 2004).

Produtos a base de extratos vegetais têm se apresentado como uma alternativa principal, ou complementar, de controle contra patógenos de plantas, devido à sua atividade antimicrobiana, não fitotóxica, sistêmica e biodegradável (Karim et al., 2016).

No processo de carbonização da madeira, obtém-se, como produto principal, o carvão vegetal. Os gases liberados neste processo normalmente são desperdiçados na atmosfera, constituindo-se em poluente (Porto et al., 2007).

O Brasil é o maior produtor de carvão vegetal do mundo, porém, poucas, ou a grande maioria das carbonizações, são efetuadas sem a recuperação dos gases. Com a condensação e recuperação dos gases voláteis obtém-se, além de carvão vegetal, o extrato pirolenhoso (líquido, ácido, licor pirolenhoso ou vinagre de madeira) e alcatrão. No Japão, a prática da utilização desses produtos na agricultura é conhecida já há muito tempo (Porto et al., 2007).

Ainda são escassas as informações científicas quanto à utilização de extrato pirolenhoso no combate às pragas e doenças, bem como, as concentrações ideais a serem utilizadas. No entanto, alguns trabalhos desenvolvidos por Miyasaka et al. (2001), Zanetti et al. (2003) e Alves (2006) vêm contribuindo com dados importantes quanto a sua utilização na agricultura.

Ao serem avaliados oito extratos orgânicos de Citrus spp., Karim et al. (2016), relataram que todos apresentaram efeito inibitório no crescimento micelial de G. citri-aurantii, indicando que os extratos da planta contêm compostos ativos que são responsáveis, pelo menos em parte, pela atividade antifúngica. Karin et al. (2017) ao testarem in vivo os extratos aquosos de Citrus populifolius e C.creticus verificaram a redução da incidência de podridão azeda em $8,33 \%$ nos frutos, quando comparados com o tratamento controle (sem tratamento). Talibi et al. (2012) relataram que extratos metanólicos de Cistus villosus, Halimium umbellatum, Ceratonia siliqua, Rubus ulmifolius, H. antiatlanticum e Pistacia atlântica apresentaram maior atividade antifúngica contra G. citri- aurantii.

Ainda dentro de estratégias de controle para a podridão azeda em frutos cítricos, a utilização de óleos essenciais tem sido uma alternativa promissora. Zhou et al. (2014a) ao estudarem o potencial de atividade de três compostos voláteis (citral, octanal e a-terpineol), em diferentes concentrações contra G. citri-aurantii, verificaram que a permeabilidade da membrana de G. citri-aurantii aumentou com concentrações crescentes dos três compostos voláteis, evidenciado pela liberação de constituintes celulares, condutividade extracelular e pH. Além disso, os compostos voláteis induziram uma diminuição no conteúdo lipídico total das células do fitopatógeno, indicando a destruição das estruturas da membrana celular.

Ruiz et al. (2016) apresentaram o primeiro relato do extrato etanólico de Parastrephia lepidophylla (Wedd.), uma espécie vegetal que cresce no noroeste argentino, com atividade contra os fungos $P$. digitatum e G. citri-aurantii em frutos de limão. $\mathrm{O}$ extrato afetou, principalmente, a esporulação e inibiu $100 \%$ a germinação dos esporos, quando se utilizou a concentração de $200 \mathrm{mg} / \mathrm{ml}$ do extrato etanólico. Segundo os autores, o possível mecanismo de ação está relacionado com a ruptura das membranas plasmáticas dos fungos. Nos testes in vivo, os autores relataram que a melhor concentração do extrato etanólico de P. leidophylla foi de $700 \mathrm{mg} / \mathrm{ml}$, com diminuição da incidência do bolor verde em frutos inoculados artificialmente.

Em outro estudo, Rodríguez et al. (2017) purificaram e caracterizaram bioquimicamente uma enzima poligalacturonase extracelular (EC 3.2.1.15), denominada PG-543, a partir de uma cultura do fungo G. citri-aurantii IEV 543, previamente isolado de fruto de limão infectado. A enzima PG-543 pode ser um fator de virulência muito importante de G. citri-aurantii, que permite e facilita a infecção no tecido do fruto. Os autores avaliaram, também, o efeito de uma proteína denominada PGIP, isolada do albedo de limão e, verificaram o efeito inibitório sobre a poligalacturonase (PG), em uma ampla gama de $\mathrm{pH}$ e temperatura, não apresentando efeito mutagênico e fitotóxico.

O uso de produtos naturais é uma alternativa de controle complementar devido à sua atividade antifúngica, à sua ação não toxica, à sua sistematicidade e biodegradabilidade (Tripathi \& Dubey 2004; Ameziane et al. 2007; 
Gatto et al. 2011). Algumas moléculas, presentes em defensivos naturais, apresentam uma estrutura química muito complexa, o que torna mais difícil para os patógenos adquirirem qualquer tipo de resistência ao produto, dando uma certa vantagem em relação à alguns princípios ativos de fungicidas. Por outro, a rápida degradação e a baixa quantidade de informações quanto ao manuseio, aos efeitos secundários e a variabilidade de efeitos podem limitar o seu uso (Cunico et al. 2003; Shabana et al., 2017).

\section{Termoterapia}

A termoterapia é uma metodologia utilizada há bastante tempo e vem despertando interesse por ser livre de qualquer resíduo, quando aplicado sobre os alimentos. Além disso, a eficácia do tratamento térmico sobre o fitopatógeno é frequentemente avaliada pela redução da viabilidade de propágulos. Estudos com a finalidade de proteger frutos com injurias pelo frio utilizam o tratamento hidrotérmico antes da refrigeração, na forma de condicionamento ou, durante o armazenamento refrigerado, na forma de aquecimento intermitente. $\mathrm{O}$ condicionamento térmico consiste em expor as frutas a temperaturas moderadas $\left(15 \mathrm{a} 25^{\circ} \mathrm{C}\right)$ ou elevadas $\left(37\right.$ a $\left.53^{\circ} \mathrm{C}\right)$, por curtos períodos, antes de refrigerá-los. Esse tratamento tem reduzido danos causados pelas baixas temperaturas e diminuindo a incidência de podridões em frutas cítricas (Jomori, 2005; Schirra \& Mulas, 1993).

A eficácia no controle de distúrbios fisiológicos pelo frio com a aplicação do aquecimento intermitente já foi verificada em algumas frutas cítricas (Felício, 2005; Jomori, 2005). Os tratamentos com temperaturas elevadas $\left(37\right.$ a $60^{\circ} \mathrm{C}$ ) têm demonstrado resultados eficientes, especialmente, no controle de doenças pós-colheita. Palou et al. (2001) ao imergirem frutos de laranjas Navelate em água nas temperaturas de 50 e $60{ }^{\circ} \mathrm{C}$, apresentaram controle de 90 a $100 \%$ do bolor azul, após os frutos serem armazenados por sete dias a $20^{\circ} \mathrm{C}$. Frutos cítricos orgânicos tratados a $56{ }^{\circ} \mathrm{C}$ por $20 \mathrm{~s}$, com simultânea escovação, reduziu a incidência de bolor verde (P. digitatum) em 50\% (Porat et al., 2000). Forner et al. (2013) observaram que o tratamento térmico de laranjas por imersão dos frutos $\left(52{ }^{\circ} \mathrm{C} / 2 \mathrm{~min}\right.$ ) reduziu a severidade do bolor verde em frutos de laranja Pera. Zhou et al. (2014b) verificaram que a imersão de frutos em água quente a $53{ }^{\circ} \mathrm{C}$ por $2 \mathrm{~min}$, provocou reduções do bolor azul e do bolor verde em frutas cítricas tratada de forma combinada com a levedura Pichia membranaefaciens, apresentando diâmetros da lesão menores do que em frutos tratados com a levedura sozinha, após 9 dias de armazenamento a $20^{\circ} \mathrm{C}$.

Segundo alguns autores, o tratamento térmico realizado antes do armazenamento é uma alternativa aos fungicidas, tanto na inibição do crescimento do patógeno, como na ativação dos mecanismos de resistência do fruto, podendo induzir a síntese de compostos antifúngicos, como as fitoalexinas ou proteínas relacionadas à patogênese (Nafussi et al., 2001; Smilanick et al., 2003; Palou et al., 2008; Forner et al., 2013).

Para Jomori (2005), os efeitos do tratamento térmico sobre a fisiologia, bioquímica e a qualidade dos frutos são poucos conhecidos e, uma vez estabelecidos, podem ajudar a identificar processos metabólicos passíveis de manipulação, proporcionando a criação de tecnologias de armazenamento que permitam o aumento da vida útil dos frutos. No entanto, alguns autores relatam o efeito desses tratamentos creditado ao estímulo na síntese de poliaminas (putrescina, espermidina e espemina), etileno e da atividade de enzimas antioxidantes (removedoras de radicais livres) (Jomori, 2005; Valero et al., 2002).

De acordo com Benato et al. (2018), a combinação da termoterapia seguida de aspersão de óleo de canela reduziu em $40,5 \%$ a severidade do bolor verde em frutos cítricos. Em estudos realizados no Laboratório de Fitopatologia e Controle Biológico do Centro Avançado de Pesquisa de Citros Sylvio Moreira (dados não publicados), o tratamento térmico $\left(52{ }^{\circ} \mathrm{C} /\right.$ três minutos $)$, combinado ou não com o método biológico (Sporobolomyces koalae), ou produto natural (quitosana a $2 \%$ por três minutos), não apresentou controle efetivo contra a podridão azeda, quando aplicado em frutos cítricos.

Por outro lado, a combinação do tratamento térmico $\left(52{ }^{\circ} \mathrm{C} / 2 \mathrm{~min}\right)$ com os isolados de levedura ACB-K1 e ACB-CR1 (Saccharomyces cerevisiae) aumentou a eficiência de controle de $P$. digitatum em frutos de lima ácida Tahiti e tangor Murcott, respectivamente (Moretto et al., 2014).

Shirra (2005) verificou controle de bolor verde em frutos cítricos, quando se combinou os produtos químicos imazalil e/ou thiabendazole nas doses de $50 \mathrm{mg} \mathrm{L}^{-1}$ com o tratamento térmico $\left(50^{\circ} \mathrm{C}\right)$. $\mathrm{O}$ autor evidencia a importância prática e sustentável desses resultados, uma vez que, doses dos fungicidas poderiam ser reduzidas durante o beneficiamento dos frutos. Conway et al. (2004) comprovaram o efeito sinérgico do tratamento térmico em combinação com leveduras antagonistas e com o bicarbonato de sódio contra Colletotrichum acutatum e Penicillium expansum, durante a fase de armazenamento de maçãs. 
A termoterapia como método de controle de doenças na pós-colheita têm suas vantagens pela ausência total de resíduos no produto tratado e o baixo impacto ao meio ambiente (Benato et al., 2018). No entanto, existem algumas limitações quanto à esse método de controle, primeiro, não controlam totalmente as doenças de pós-colheita, sendo necessário a combinação com outras estratégias, como por exemplo o uso de produtos biológico ou químicos, sendo portanto, considerado mais uma alternativa complementar de controle das podridões. Em segundo lugar, de acordo com alguns autores, a termoterapia pode afetar a qualidade dos frutos (Usall et al., 2016; Palou et al., 2001).

\section{CONCLUSÃO}

As informações apresentadas neste estudo demonstram a potencialidade de métodos alternativos, combinados ou não entre si, para o controle de doenças que ocorrem na fase pós-colheita de citros. No entanto, vários desafios ainda precisam ser superados para que uma nova tecnologia possa ser empregada durante o beneficiamento dos frutos cítricos, a fim de se desenvolver uma estratégia comercialmente viável que possa atender produtores orgânicos e convencionais.

\section{AGRADECIMENTOS}

A CAPES (Agência Federal de Apoio e Avaliação do Ensino de Pós-Graduação) pela concessão da bolsa de estudos para Vanessa Santos Moura.

\section{REFERÊNCIAS}

Ait Barka, E., Eullaffroy, P., Clement, C., \& Vernet, G. (2004). Chitosan improves development, and protects Vitis vinifera L. against Botrytis cinerea. Plant Cell Reports, 22, 608-614.

Alves, M. (2006) Impacto da utilização de fino de carvão e extrato pirolenhoso na agricultura (Dissertação de mestrado). Universidade Estadual Paulista "Júlio de Mesquita Filho", Jaboticabal.

Ameziane, N., Boubaker, H., Boudyach, E. H., Msanda, F., Jilal, A., \& Ait Benaoumar, A. (2007). Antifungal activity of Moroccan plants against citrus fruit pathogens. Agronomy for Sustainable Development, 27, 273-277.
Bautista-Banõs, S., Hernandez-Lauzardo, A. N., Velazquez-Del Valle, M. G., Hernández-Lopez, M., Barka, E. A., Bosquez-Molina, E., \& Wilson, C. L. (2006). Chitosan as a potential natural compound to control pre and postharvest diseases of horticultural commodities. Crop Protection, 25, 108-118.

Benato, E. A., Belletti, T. C., Terao, D., \& Franco, D. A. S. (2018). Óleos essenciais e tratamento térmico no controle pós-colheita de bolor verde em laranja. Summa Phytopathologica, 44(1), 65-71.

Benato, E. A. (1999). Controle de doenças pós-colheita em frutas tropicais. Summa Phytopathologica, 25(1), 90-93.

Cappellini, R. A., \& Ceponis, M. J. (1984) Postharvest losses in fresh fruits and vegetables: postharvest losses in perishable crops. In: H. E. Moline (Ed.), Postharvest pathology of fruits and vegetables: postharvest losses in perishable crops (pp. 24-30). Berkeley: University of California Agricultural Experiment Station.

Carmona-Hernandez, S., Reyes-Pérez, J. J., ChiquitoContreras, R. G., Rincon-Enriquez, G., Cerdan-Cabrera, C. R., \& Hernandez-Montiel, L. G. (2019). Biocontrol of postharvest fruit fungal diseases by bacterial antagonists: A review. Agronomy, 9(3), 121.

Cheah, L. H., Page, B. B. C., \& Sheperd, R. (1997). Chitosan coating for inhibition of Sclerotina carrots. New Zealand Journal of Crop and Horticultural Science, 25, 89-92.

Coelho, A. R., Hoffmann, F. L., \& Hirooka, E. Y. (2003). Biocontrol by yeasts: perspectives in aplication for fruits safety: a review. Semina: Ciências Agrárias, 24, 337-358.

Conway, W. S., Leverents, B., Janisiewicz, W. J., Blodgett, A. B., Saftner, R. A., \& Camp, M. J. (2004). Integranting heat treatment, biocontrol and sodium bicarbonate to reduce postharvest decay of strawberry fruit. Journal of Agricultural and Food Chemistry, 45, 11-20.

Cunha, T., Ferraz, L. P., Wehr, P. P., \& Kupper, K. C. (2018). Antifungal activity and action mechanisms of yeasts isolates from citrus against Penicillium italicum. International Journal of Food Microbiology, 276, 20-27.

Cunico, M. M., Miguel, O. G., Miguel, M. D., Carvalho, J. L. S., Peitz, C., Auer, C. G., \& Grigoletti Júnior, A. (2003). Estudo da atividade antifúngica 396 de Ottonia martiana Miq., Piperaceae: um teste in vivo. Visão Acadêmica, 4(2), 77-82.

Droby, S., Wisniewski, M., Macarisin, D., \& Wilson, C. (2009). Twenty years of postharvest biocontrol research: 
Is it time for a new paradigm? Postharvest Biology and Technology, 52, 137-145.

Droby, S., Vinokur, V., Weiss, B., Cohen, L., Daus, A., Goldschmidt, E., \& Porat, R. (2002). Induction of resistance to Penicillium digitatum in grapefruit by the yeast biocontrol agent Candida oleophila. Phytopathology, 92, 393-399.

Dukare, A. S., Sangeeta, P., Nambi, V. E., Gupta, R., Sharma, K., \& Vishwakarma, R. K. (2018). Exploitation of microbial antagonists for the control of postharvest diseases of fruits: a review. Critical Reviews in Food Science and Nutrition, 16, 1-16.

Dutta, P. K., Tripathi, S., Mehrotra, G. K., \& Dutta, J. (2007). Perspectives for chitosan based antimicrobial films in food applications. Food Chemistry, 114, 1173-1182.

El-Ghaouth, A., Arul, J., Asselin, A., \& Benhamou, N. (1992). Antifungal activity of chitosan on post-harvest pathogens: induction of morphological and cytological alterations in Rhizopus stolonifer. Mycological Research, 96, 769-779.

El-Ghaouth, A., Smilanick, J. L., Brown, G. E., Wisniewski, M., \& Wilson, C. L. (1999). Application of Candida saitoana and glycolchitosan for the control of postharvest diseases of apple and citrus fruit under semi-commercial conditions. Plant Disease, 84, 243-248.

El-Ghaouth, A., Smilanick, J. L., \& Wilson, C. L. (2000). Enhancement of the performance of Candida saitoana by the addition of glycochitosan for control of postharvest decay of apple and citrus fruit. Postharvest Biology and Technology, 19, 249-253.

Felício, A. H. (2005). Conservação refrigerada de tangor 'Murcott' tratada termicamente (Tese de doutorado). Universidade de São Paulo, Piracicaba.

Feng, L., Wu, F., Li, J., Jiang, Y., \& Duan, X. (2011). Antifungal activities of polyhexamethylene biguanide and polyhexamethylene guanide against the citrus sour rot pathogen Geotrichum citri-aurantii in vitro and in vivo. Postharvest Biology and Technology, 61, 160-164.

Ferraz, L. P., Cunha, T., Silva, A. C. \& Kupper, K. C. (2016). Biocontrol ability and putative mode of action of yeast against Geotrichum citri-aurantii in citrus fruit. Microbiological Research, 188, 72-79.

Filonow, A. B. (1998). Role of competition for sugars by yeasts in the biocontrol of gray mould of apple. Biocontrol Science and Technology, 8, 243-256.
Forner, C., Bettiol, W., Nascimento, L. M., \& Terao, D. (2013). Controle em pós-colheita de Penicillium digitatum em laranja-pera com microrganismos e tratamento térmico. Revista Brasileira de Fruticultura, 35(1), 23-31.

Fujimoto A. \& Kupper K. C. (2016). Production of Antifungal Compounds and Hydrolytic Enzymes by Bacillus spp. As Mechanisms of Action against Phyllosticta citricarpa. IOSR Journal of Agriculture and Veterinary Science, 9(11), 19-27.

Gatto, M. A., Ippolito, A., Linsalata, V., Cascarano, N. A., Nigro, F., Vanadia, S., \& Di Venere, D. (2011). Activity of extracts from wild edible herbs against postharvest fungal diseases of fruit and vegetables. Postharvest Biology and Technology, 61, 72-82.

Hao, W., Zhong, G., Hu, M., Luo, J., Weng, Q., \& Rizwanul-Haq, M. (2010). Control of citrus postharvest green and blue mold and sour rot by tea saponin combined with imazalil and prochloraz. Postharvest Biology and Technology, 56, 39-43.

Hong, P., Hao, W., Luo, J., Chen, S., Hu, M., \& Zhong, G. (2014). Combination of hot water, Bacillus amyloliquefaciens HF-01 and sodium bicarbonate treatments to control postharvest decay of Mandarin fruit. Postharvest Biology and Technology, 88, 96-102.

Ismail, M., \& Zhang, J. (2004). Post-harvest citrus diseases and their control. Outlooks on Pest Management, 15, 29-35.

Johnson, G. I., \& Heather, N. W. (1995). Postharvest disease and pest control in tropical fruit. In B. R. Champ, \& E. Highley (Eds.), Postharvest technology for agricultural products in Vietnam (Proceedings ${ }^{\circ} 60, \mathrm{pp} .100-126$ ). Canberra: ACIAR.

Jomori, M. L. L. (2005). Resistência de lima ácida 'Tahiti à baixa temperatura: tratamento térmico e envolvimento do etileno (Tese de doutorado). Universidade de São Paulo, Piracicaba.

Junior, G. M. B., Junior, A. F. C., Chagas, L. F. B., Carvalho Filho, M. R., Oliveira Miller, L. \& Santos, G. R. (2017). Controle biológico de fitopatógenos por Bacillus subtilis in vitro. Biota Amazônia, 7(3), 45-51.

Karim, H., Boubaker, H., Askarne, L., Talibi, I., Msanda, F., Boudyach, E. H., Saadi, B., \& Ait Ben Aoumar, A. (2016). Antifungal properties of organic extracts of eight Cistus L. species against postharvest citrus sour rot. Letters in Applied Microbiology, 1, 16-22. 
Karin, H., Boubaker, H., Askarne, L., Cherifi, K., Lakhtar, H., Msanda, F., Boudyach, E. H., \& Ait Ben Aoumar, A. (2017). Use of Cistus aqueous extracts as botanical fungicides in the control of Citrus sour rot. Microbial Pathogenesis, 104, 263-267.

Kellerman, M., Liebenberg, E., Njombolwana, N., Erasmus, A., \& Fourie, P. H. (2018). Postharvest dip, drench and wax coating application of pyrimethanil on citrus fruit: Residue loading and green mould control. Crop Protection (Guildford, Surrey), 103, 115-129.

Klein, M. N., \& Kupper, K. C. (2018). Biofilm production by Aureobasidium pullulans improves biocontrol against sour rot in citrus. Food Microbiology, 69, 1-10.

Kupper, K. C., Cervantes, A. L. L., Klein, M. N., \& Silva, A. C. (2013). Evaluation of antagonistic microorganisms. Saccharomyces cerevisiae and Bacillus subtilis for control of Penicillium digitatum. Revista Brasileira de Fruticultura, 35, 425-436.

Kupper, K. C., \& Fernandes, N. G. (2002). Isolamento e seleção de Bacillus spp. para o controle de Colletotrichum acutatum em flores destacadas de lima ácida 'Tahiti'. Summa Phytopathologica, 28(3), 292-295.

Kupper, K. C., Ferraz, L. P., da Silva, A. C., \& Coletta Filho, H. D. (2016). Doenças dos citros. Informe Agropecuário, 37, 36-53.

Kurtzman, C. P., \& Droby, S. (2001). Metschnikowia fructicola, a new ascosporic yeast effective for biocontrol of postharvest fruit rots. Systematic and Applied Microbiology, 24, 395-399.

Lahlali, R., Raffaele, B., \& Jijakli, M. H. (2011). UV protectants for Candida oleophila (strain O), a biocontrol agent of postharvest fruit diseases. Plant Pathology, 60, 288-295.

Leelasuphakul, W., Hemmanee, P., \& Chuenchitt, S. (2008). Growth inhibitory properties of Bacillus subtilis strains and their metabolites against the green mold pathogen (Penicillium digitatum Sacc.) of citrus fruit. Postharvest Biology and Technology, 48(1), 113-121.

Liu, J., Sui, Y., Wisniewski, M., Droby, S., \& Liu, Y. (2013). Review: utilization of antagonistic yeasts to manage postharvest fungal diseases of fruit. International Journal of Food Microbiology, 167, 153-160.

Liu, X., Wang, L. P., Li, Y. C., Li, H. Y., Yu, T., \& Zheng, X. D. (2009). Antifungal activity of thyme oil against
Geotrichum citri-aurantii in vitro and in vivo. Journal of Applied Microbiology, 107, 1450-1456.

Lopes, R. B. (2009) A indústria no controle biológico: produção e comercialização de microrganismos no Brasil. In W. Bettiol \& M. A. B. Morandi (Eds.), Biocontrole de doenças de plantas: uso e perspectiva. (pp. 15-28). Jaguariúna: Embrapa Meio Ambiente.

Luo, J., Xia, W., Cao, P., Xiao, Z. A., Zhang, Y., Liu, M., Zhan, C., \& Wang, N. (2019). Integrated transcriptome analysis reveals plant hormones jasmonic acid and salicylic acid coordinate growth and defense responses upon fungal infection in poplar. Biomolecules, 9(1), 12. Maia, C. C. P. (2011). Produção de um agente de controlo biológico recorrendo a subprodutos da indústria agroalimentar (Dissertação de mestrado). Faculdade de Ciências e Tecnologia, Universidade do Algarve, Portugal.

McKay, A. H., Foster, H., \& Adaskaveg, J. (2012). Efficacy and application strategies for propiconazole as a new postharvest fungicide for managing sour rot and green mold of citrus fruit. Plant Disease, 96, 235-242.

Medina-Córdova, N., Rosales-Mendoza, S., HernandezMontiel, L. G., \& Angulo, C. (2018). The potential use of Debaryomyces hansenii for the biological control of pathogenic fungi in food. Biological Control, 121, 216-222.

Miyasaka, S., Ohkawara, T., \& Kunio, N. (2001) Derivados de carvão vegetal, extrato pirolenhoso e fino de carvão na agricultura natural: apostila. Miyazaki: APAN.

Moretto, C., Cervantes, A. L. L., Batista Filho, A., \& Kupper, K. C. (2014). Integrated control of green mold to reduce chemical treatment in post-harvest citrus fruits. Scientia Horticulturae, 165, 433-438.

Nafussi, B., Ben-Yehoshua, S., Rodov, V., Peretz, J., Ozer, B. K., \& D'Hallewin, G. (2001). Mode of action of hot-water dip in reducing decay of lemon fruit. Journal of Agricultural and Food Chemistry, 49(1), 107-113.

Neves, M. F., \& Trombin, V. G. (2017). Anuário da citricultura (1. ed.). São Paulo: Citrusbr.

Nunes, C. A. (2012). Biological control of postharvest diseases of fruit. European Journal of Plant Pathology, 133, 181-196.

Obagwu, J., \& Korsten, L. (2003). Control of citrus green and blue molds with garlic extracts. European Journal of Plant Pathology, 109, 221-225. 
Palou, L., Smilanick, J. L., \& Droby, S. (2008). Alternatives to conventional fungicides for the control of citrus postharvest green and blue moulds. Stewart Postharvest Review, 4, 1-16.

Palou, L., Smilanick, J. L., Usall, J., \& Viñas, I. (2001). Control of postharvest blue and green molds of oranges by hot water, sodium carbonate, and sodium bicarbonate. Plant Disease, 85, 371-376.

Pinchuk, I. V., Bressollier, P., Sorokulova, I. B., Verneuil, B., \& Urdaci, M. C. (2002). Amicoumacin antibiotic production and genetic diversity of Bacillus subtillis strain isolated from different habitats. Research in Microbiology, 153, 269-276.

Pitt, J. I., \& Hocking, A. D. (1997). Fungi and Food Spoilage (2nd ed.). Maryland: Aspen Publishiers Inc.

Porat, R., Daus, A., Weiss, B., Cohen, L., Fallik, E., \& Droby, S. (2000). Reduction of postharvest decay in organic citrus fruit by a short hot water brushing treatment. Postharvest Biology and Technology, 18, 151-157.

Porto, P. R., Sakita, A. E. N., \& Sakita, M. N. (2007). Efeito da aplicação do extrato pirolenhoso na germinação e no desenvolvimento de mudas de Pinus elliottii var. elliottii. IF Série Registros, 31, 15-19.

Rodríguez, I. F., Torres, S., Sayago, J. E., Zampini, I. C., Isla, M. I., \& Ordóñez, R. (2017). Inhibition of an extracellular polygalacturonase from geotrichum candidum by a proteinaceous inhibitor isolated from lemon fruits. Journal of Microbiology, Biotechnology and Food Sciences, 6, 1019-1025.

Romanazzi, G., Feliziani, E., Santini, M., \& Landi, L. (2013). Effectiveness of postharvest treatment with chitosan and other resistance inducers in the control of storage decay of strawberry. Postharvest Biology and Technology, 75, 24-27.

Ruiz, M. D. P., Ordóñeza, R. M., María, I. I., \& Sayagoa, J. E. (2016). Activity and mode of action of Parastrephia lepidophylla ethanolic extracts on phytopathogenic fungus strains of lemon fruit from Argentine Northwest. Postharvest Biology and Technology, 114, 62-68.

Sanders, M. E., Morelli, L., \& Tompkins, T. A. (2003). Sporeformers as human probiotics: Bacillus, Sporolactobacillus, and Brevibacillus. Comprehensive Reviews in Food Science and Food Safety, 2(3), 101-110.
Schirra, M., \& Mulas, M. (1993). Keeping quality of "Oroblanco" grapefruit-type as affected by hot dip treatments. Advances in Horticultural Science, 7, 73-76.

Senhor, R. F., Souza, P. A., Andrade Neto, R. C., Maracajá, P. B. \& Nascimento, F. J. (2009) Manejo de doenças póscolheita. Revista Verde de agroecologia e desenvolvimento sustentável, 4(1), 1-13.

Shabana, Y. M., Abdalla, M. E., Shahin, A. A., El-Sawy, M. M., Draz, I. S., \& Youssif, A. W. (2017). Efficacy of plant extracts in controlling wheat leaf rust disease caused by Puccinia triticina. Egyptian Journal of Basic and Applied Sciences, 4, 67-73.

Shirra, M. (2005). Postharvest pest management of horticultural crops by combined heat therapy and fungicide treatment. Acta Horticulturae, (682), 2127-2132.

Smilanick, J. L., Sorenson, D., Mansour, M., Aieyabei, J., \& Plaza, P. (2003). Impact of a brief postharvest hot water drench treatment on decay, fruit appearance, and microbe populations of California lemons and oranges. HortTechnology, 13(2), 333-338.

Spadaro, D., \& Droby, S. (2016). Development of biocontrol products for postharvest diseases of fruit: the importance of elucidating the mechanisms of action of yeast antagonists. Trends in Food Science \& Technology, 47, 39-49.

Talibi, I., Askarne, L., Boubaker, H., Boudyach, E. H., Msanda, F., Saadi, B., \& Ait Ben Aoumar, A. (2012). Antifungal activity of some Moroccan plants against Geotrichum candidum, the causal agent of postharvest citrus sour rot. Crop Protection (Guildford, Surrey), 35, 41-46.

Terry, L. A., \& Joyce, D. C. (2003). Elicitors of induced disease resistance in postharvest horticultural crops: a brief review. Postharvest Biology and Technology, 32, 1-13.

Tripathi, P., \& Dubey, N. K. (2004). Exploitation of natural products as an alternative strategy to control postharvest fungal rotting of fruit and vegetables. Postharvest Biology and Technology, 32, 235-245.

Usall, J., Teixidó, N., Abadias, M., Torres, R., Cañamas, T., \& Viñas, I. (2009). Improving formulation of biocontrol agents manipulating production process. In D. Prusky \& M. L. Gullino (Eds.), Postharvest Pathology (pp. 149169). Dordrecht: Springer.

Usall, J., Ippolito, A., Sisquella, M., \& Neri, F. (2016). Physical treatments to control postharvest diseases of 
fresh fruits and vegetables. Postharvest Biology and Technology, 122, 30-40.

Valero, D., Martínez-Romero, D. \& Serrano, M. (2002). The role of polyamines in the improvement of the shelf life of fruit. Trends in Food Science \& Technology, 13(6), 228-234.

Wagner, A., Hetman, B., Kopacki, M., Jamiołkowska, A., Krawiec, P., \& Lipa, T. (2013). Laboratory effect of Boni Protect containing Aureobasidium pullulans (de Bary) Arnoud in the control of some fungal diseases of apple fruit. Acta Agrobotanica, 66, 77-88.

Zambolim, L. (2002). Controle integrado de doenças em pós-colheita de frutíferas tropicais. In: Anais do Simpósio de controle de doenças de plantas (pp. 139-182). Lavras, NEFIT/UFLA.

Zambolim, L., Cista, H., Ventura, J. A., \& Vale, F. X. R. (2002). Controle de doenças em póscolheita de frutas tropicais. In L. Zambolim (Ed.), Manejo integrado: fruteiras tropicais - doenças e pragas (pp. 443-512). Viçosa: UFV.

Zanetti, M., Cazetta, J. O., Mattos Júnior, D., \& Carvalho, A. S. (2003). Uso de subprodutos de carvão vegetal na formação do porta-enxerto limoeiro cravo em ambiente protegido. Revista Brasileira de Fruticultura, 25, 1-8.
Zhimo, V. Y., Bhutia, D. D., \& Saha, J. (2016). Biological control of post harvest fruit diseases using antagonistic yeasts in India. Journal of Plant Pathology, 98(2), 275-283.

Zhou, H., Tao, N., \& Jia, L. (2014a). Antifungal activity of citral, octanal and $\alpha$-terpineol against Geotrichum citri-aurantii. Food Control, 37, 277-283.

Zhou, Y., Deng, L., \& Zeng, K. (2014b). Enhancement of biocontrol efficacy of Pichia membranaefaciens by hot water treatment in postharvest diseases of citrus fruit. Crop Protection, 63, 89-96.

Zulian,A., Dörr,A. C., \& Almeida, S. C. (2013). Citricultura e agronegócio cooperativo no Brasil. Revista Eletrônica em Gestão. Educação e Tecnologica Ambiental, 11, 2290-2306.

Recebido: Junho 14, 2018

Aceito: Abril 01, 2019

Como citar: Moura, V. S., Moretto, R. K., Machado, B. I. \& Kupper, K. C. (2019). Alternativas de controle de doenças pós-colheita de citros. Citrus Research \& Technology, 40, e1044. https://doi.org/10.4322/crt.17819. 